\title{
Características agronômicas e tecnológicas de genótipos de feijão do grupo comercial Carioca
}

\author{
Leandro Borges Lemos ${ }^{(1)}$, Ricardo Soares de Oliveira ${ }^{(1)}$, Edwin Camacho Palomino(1) \\ e Tiago Roque Benetoli da Silva(1)
}

(1)Universidade do Estado de São Paulo (Unesp), Faculdade de Ciências Agronômicas (FCA), Dep. de Produção Vegetal, Caixa Postal 237, CEP 18603-970 Botucatu, SP. E-mail: leandrobl@fca.unesp.br, rsoliveira@fca.unesp.br, palomino@fca.unesp.br, benetoli@fca.unesp.br

\begin{abstract}
Resumo - A busca por cultivares produtivas, adaptadas ao local de cultivo e com características tecnológicas desejáveis é uma constante. O objetivo deste trabalho foi avaliar o comportamento de genótipos de feijão, do grupo comercial Carioca, quanto a características agronômicas e tecnológicas. Vinte e nove genótipos foram cultivados na época das águas, nos anos de 2001 e 2002, e distribuídos em blocos casualizados, com quatro repetições. Sobressaíram-se os genótipos IAC-Carioca, FT-Bonito, Rudá, Porto Real, CNFC 8008, CNFC 8011, CNFC 8012, CNFC 8013 e CNFC 8156 com produtividade de grãos acima da média obtida. Destacaram-se com produtividade média de grãos acima de $3.000 \mathrm{~kg} \mathrm{ha}^{-1}$ e tempo de cozimento médio em torno de 20 minutos, os genótipos IAC-Carioca, CNFC 8012 e CNFC 8156.
\end{abstract}

Termos para indexação: Phaseolus vulgaris, produtividade, componentes da produção, proteína bruta, hidratação dos grãos.

\section{Agronomic and technologic characteristics of common bean genotypes from Carioca commercial group}

\begin{abstract}
Cultivars with high yield, adaptability and desirable technological characteristics are a must. The objective of this work was to evaluate agronomic and technologic characters of common bean genotypes from carioca commercial group. Genotypes were cultivated in water growing season, in 2001 and 2002. The experimental design was by randomized blocks with 29 genotypes and four replications. The IAC-Carioca, FT-Bonito, Rudá, Porto Real, CNFC 8008, CNFC 8011, CNFC 8012, CNFC 8013 and CNFC 8156 genotypes yielded above the average. The genotypes IAC-Carioca, CNFC 8012 and CNFC 8156 presented the best results with 3,000 kg ha-1 and 20 minutes cooking time.
\end{abstract}

Index terms: Phaseolus vulgaris, yield, production components, crude protein, grain hydration.

\section{Introdução}

A melhoria do desempenho produtivo da cultura do feijão comum (Phaseolus vulgaris L.) associada à obtenção de novas cultivares com características agronômicas desejáveis vem aumentando com o passar do tempo, o que evidencia maior preocupação com a interação entre genótipos e ambientes, com as diferenças no comportamento das linhagens e das cultivares, em diversos locais, anos agrícolas e épocas de semeadura (Duarte \& Zimmermann, 1994; Carbonell \& Pompeu, 2000; Carbonell et al., 2001).

Os programas brasileiros para o melhoramento genético do feijoeiro têm dado maior ênfase à obtenção de cultivares do grupo comercial Carioca pela grande demanda do mercado (Zimmermann et al., 1996).
No Brasil, na década de 90, foram colhidas, em média, 3,5 milhões de toneladas por ano, das quais 2,7 milhões são de feijão comum, com 1,6 milhões do tipo Carioca, 0,6 milhões do grupo comercial preto e 0,5 milhões restantes de outros tipos (Ferreira et al., 2002).

$\mathrm{O}$ consumo de feijão vem diminuindo nos últimos 40 anos, ou seja, de mais de $20 \mathrm{~kg} \mathrm{hab}^{-1} \mathrm{ano}^{-1}$ na década de 70 , passou para $16 \mathrm{~kg} \mathrm{hab}^{-1} \mathrm{ano}^{-1}$, no final dos anos 90, representando uma redução de $1,3 \%$ ao ano, enquanto a população cresceu 2,2\% (Yokoyama et al., 1996; Ferreira et al., 2002).

Mesmo assim, o feijão ainda é um dos alimentos tradicionais da dieta do brasileiro, constituindo importante fonte protéica e energética. No entanto, é um produto que perde rapidamente o valor comercial após a colheita, por causa, principalmente, da diminuição da capaci- 
dade de reidratação, do aumento do tempo necessário de cozimento e do escurecimento do tegumento (Sawazaki et al., 1985; Bressani, 1989; Iaderoza et al., 1989).

Parâmetros que norteiam o melhoramento genético do feijoeiro são o tempo de cozimento, as características de hidratação, o conteúdo protéico e o balanço em aminoácidos de sua proteína, influenciados diretamente pelo fator genético (Durigan et al., 1978) e a interação genótipo $\mathrm{x}$ ambiente (Bressani, 1989).

O objetivo deste trabalho foi avaliar o comportamento de genótipos de feijão do grupo comercial Carioca, quanto a características agronômicas e tecnológicas.

\section{Material e Métodos}

Foram realizados dois experimentos na época das águas, nos anos de 2001 e 2002, na Fazenda Experimental da Faculdade de Ciências Agronômicas, Unesp, Campus de Botucatu, localizada no Município de São Manuel, SP, em área anteriormente ocupada com a cultura de aveia, num solo apresentando 80 e $20 \mathrm{~g} \mathrm{~kg}^{-1}$ de areia e argila, respectivamente. O local encontra-se a $22^{\circ} 45^{\prime} \mathrm{S}, 48^{\circ} 34^{\prime} \mathrm{O}$, com altitude de $750 \mathrm{~m}$. Os dados climáticos encontram-se na Figura 1.

A análise química do solo $(0-20 \mathrm{~cm})$ em 2001 revelou os seguintes resultados: $\mathrm{P}, 20 \mathrm{mg} \mathrm{dm}^{-3} ; \mathrm{MO}, 11 \mathrm{~g} \mathrm{dm}^{-3} ; \mathrm{pH}$ em $\mathrm{CaCl}_{2}, 5,1 ; \mathrm{K}, \mathrm{Ca}, \mathrm{Mg}, \mathrm{H}+\mathrm{Al}, \mathrm{SB}$ e CTC, $1,4,15,5$, 28, 21,4 e 49,4 $\mathrm{mmol}_{\mathrm{c}} \mathrm{dm}^{-3}$, respectivamente, e $43 \%$ de $\mathrm{V}$ (saturação por bases). Aplicou-se calcário em $12 / 6 / 2001$, objetivando elevar a saturação por bases a $70 \%$. O calcário foi o dolomítico com PRNT de $91 \%$ na dose de 1,5 t ha-1. No ano de 2002, a análise química do solo apresentou os seguintes valores: $\mathrm{P}, 42 \mathrm{mg} \mathrm{dm}^{-3}$; $\mathrm{MO}$, $14 \mathrm{~g} \mathrm{dm}^{-3} ; \mathrm{pH}$ em $\mathrm{CaCl}_{2}, 6,3 ; \mathrm{K}, \mathrm{Ca}, \mathrm{Mg}, \mathrm{H}+\mathrm{Al}, \mathrm{SB}$ e CTC, $2,5,29,14,15,46$ e $60 \mathrm{mmol}_{\mathrm{c}} \mathrm{dm}^{-3}$, respectivamente, e V\% igual a 76, sem necessidade de aplicação de calcário.

Aplicaram-se, nos sulcos de semeadura, $300 \mathrm{~kg} \mathrm{ha}^{-1} \mathrm{da}$ fórmula comercial 4-14-8+0,4\% de boro. Foram realizadas duas adubações de cobertura: a primeira aos 26 dias após a semeadura (DAS) e a segunda com 45 DAS, utilizando-se $70 \mathrm{~kg} \mathrm{ha}^{-1}$ da fórmula comercial 20-0-20 em cada aplicação, nos dois anos de experimentação.

As semeaduras foram realizadas manualmente em 16/8/2001 e 19/8/2002, utilizando-se 18 sementes por metro, com espaçamento entre linhas de $0,50 \mathrm{~m}$ (240.000 plantas ha $\left.{ }^{-1}\right)$.

O delineamento experimental utilizado foi o de blocos casualizados com 29 genótipos de feijoeiro do grupo comercial Carioca, com quatro repetições, com parcela experimental constituindo-se de 4 linhas de $4 \mathrm{~m}$ de comprimento, com espaços de $0,50 \mathrm{~m}$. A área útil de $3 \mathrm{~m}^{2}$ era formada pelas duas linhas centrais, eliminando-se $0,5 \mathrm{~m}$ das extremidades de cada linha.

Os genótipos de feijão utilizados foram: Carioca, Pérola, IAC-Carioca, IAC-Carioca Eté, IAC-Carioca Pyatã, IAC-Carioca Aruã, Campeão 2, Carioca Precoce, FT-Bonito, Rudá, Aporé, Princesa, IAPAR 14, IAPAR 80, IAPAR 81, Porto Real, CNFC 8005, CNFC 8006, CNFC 8007, CNFC 8008, CNFC 8009, CNFC 8010, CNFC 8011, CNFC 8012, CNFC 8013, CNFC 8156, RELAV 37-19, MA 534534 e EL 49.

As irrigações foram efetuadas utilizando-se sistema do tipo aspersão convencional, com turno de rega de cinco dias, sempre que necessário, tomando-se cuidado especial com as fases críticas da cultura como emergência de plântulas, floração e enchimento de vagens.

Foram avaliadas características agronômicas (produtividade de grãos, índice relativo de produtividade de grãos, número de vagens/planta, número de grãos/vagem e massa de 100 grãos) e características tecnológicas dos grãos (teor de proteína bruta, tempo de cozimento, capacidade de hidratação e presença de grãos de casca dura que não hidrataram).

A produtividade de grãos foi estimada em $\mathrm{kg} \mathrm{ha}^{-1}$, em razão do rendimento de grãos na área útil de cada parcela experimental e o índice relativo (\%), expresso para cada genótipo em relação às cultivares Carioca e Pérola.

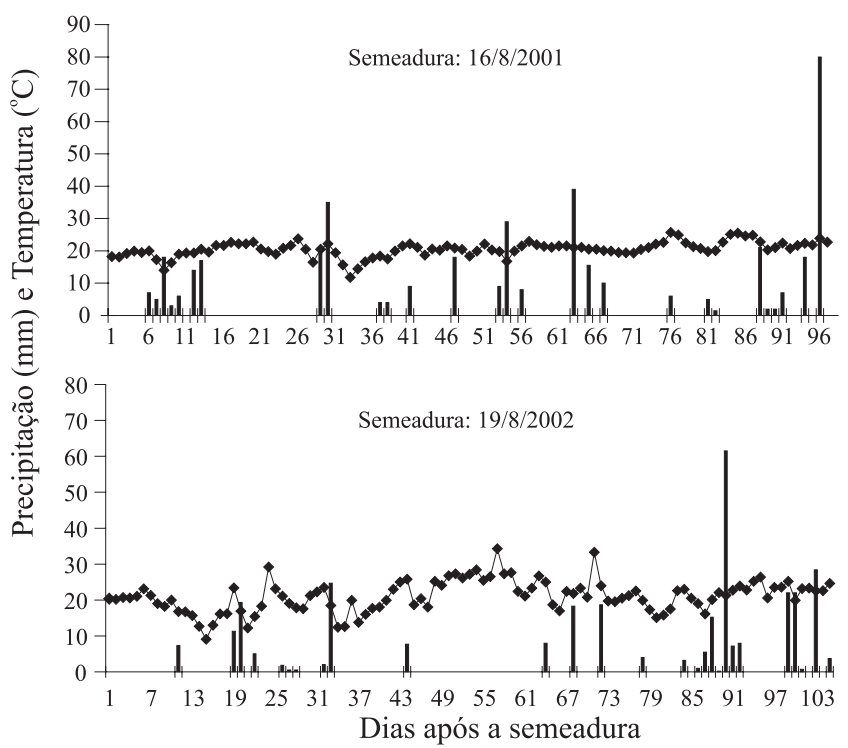

Figura 1. Precipitação pluvial (ם) e temperatura média $(\bullet)$ ocorridas em experimento de genótipos de feijão em São Manuel, SP, em 2001 e 2002. 
Para obtenção dos componentes de produção, número de vagens/planta, número de grãos/vagem e massa de 100 grãos, foram coletadas ao acaso 10 plantas na área útil de cada parcela experimental.

O teor de proteína bruta (PB\%) foi determinado pela fórmula $\mathrm{PB}=\mathrm{N}$ total $\mathrm{x} 6,25$, em que $\mathrm{N}$ total é o teor de $\mathrm{N}$ nos grãos, obtido pelo método de Kjeldahl (Sarruge \& Haag, 1974).

O tempo de cozimento (minutos) foi determinado em grãos-recém colhidos (10 dias após a colheita) com o auxílio do Cozedor de Mattson. Todas as partes do aparelho, compreendidas pelos receptáculos e estiletes, ficam dentro de água quente, mantida em nível constante; a média da temperatura da água, verificada periodicamente, foi de $96^{\circ} \mathrm{C}$, nos dois anos de experimentação (Durigan, 1979).

A capacidade de hidratação foi determinada em amostras de $50 \mathrm{~g}$ colocadas em água destilada durante 12 horas. Nas primeiras quatro horas, o volume de água foi determinado a cada 30 minutos e nas 8 horas restantes, a cada hora. Ao final do tempo previsto para a hidratação, a água foi totalmente drenada e os grãos pesados. Por meio da diferença entre o peso final e o peso inicial, obteve-se a quantidade de água absorvida na amostra. Os grãos com casca dura ("hardshell") que não hidrataram foram pesados separadamente para determinar sua porcentagem, calculada pela relação entre o peso de grãos não hidratados e o peso inicial da amostra, multiplicado por 100 (Durigan, 1979). Durante a condução do teste, verificou-se que a temperatura média da água foi de $25^{\circ} \mathrm{C}$ nos dois anos de experimentação.

Os dados foram submetidos à análise de variância, utilizando-se o teste $\mathrm{F}$, e a comparação das médias foi feita pelo teste de Tukey a 5\% de probabilidade. Realizou-se análise de regressão entre o tempo (horas) e a capacidade de hidratação $(\mathrm{mL})$, visando determinar o tempo necessário para que a máxima hidratação dos grãos dos genótipos de feijão ocorra. Em relação aos grãos com casca dura que não hidrataram, não foi realizada análise estatística.

\section{Resultados e Discussão}

A produtividade de grãos variou de $2.151 \mathrm{~kg} \mathrm{ha}^{-1} \mathrm{a}$ $3.623 \mathrm{~kg} \mathrm{ha}^{-1}$ no ano de $2001 \mathrm{e}$ de $1.449 \mathrm{~kg} \mathrm{ha}^{-1} \mathrm{a}$ $3.412 \mathrm{~kg} \mathrm{ha}^{-1}$ no ano de 2002 (Tabela 1). Carbonell et al. (2003) obtiveram produtividades acima de $3.000 \mathrm{~kg} \mathrm{ha}^{-1}$, no Estado de São Paulo na safra das águas de 2001 e
2002, e a produtividade média de dezoito genótipos de feijão foi de $2.700 \mathrm{~kg} \mathrm{ha}^{-1}$.

Em produtividade de grãos, destacaram-se no ano de 2001, os genótipos IAC-Carioca, FT-Bonito, Rudá, Princesa, IAPAR 80, IAPAR 81, Porto Real, CNFC 8005, CNFC 8006, CNFC 8007, CNFC 8008, CNFC 8010, CNFC 8011, CNFC 8012, CNFC 8013, CNFC 8156 e o RELAV 37-19, com produtividade acima da média (2.833 $\left.\mathrm{kg} \mathrm{ha}^{-1}\right)$ e índices relativos superiores aos das cultivares testemunhas Carioca e Pérola. Já os genótipos IAC-Carioca e CNFC 8010 apresentaram produtividade acima de $3.500 \mathrm{~kg} \mathrm{ha}^{-1}$, com número de vagens por planta e número de grãos por vagem, acima da média (Tabela 2). No ano de 2002, obtiveram produtividades de grãos acima da média (2.318 $\left.\mathrm{kg} \mathrm{ha}^{-1}\right)$, os genótipos Carioca, IAC-Carioca, FT-Bonito, Rudá, Porto Real, CNFC 8008, CNFC 8009, CNFC 8011, CNFC 8012, CNFC 8013, CNFC 8156 e MA 534534, todos com índices relativos acima da testemunha Pérola. Apenas os genótipos CNFC 8009, CNFC 8012 e CNFC 8156 apresentaram índices relativos acima da cultivar testemunha Carioca. Já os genótipos CNFC 8012 e CNFC 8156 tiveram produtividades acima de $3.000 \mathrm{~kg} \mathrm{ha}^{-1}$, com massa de 100 grãos e número de grãos superiores à média (Tabela 2).

Esses resultados mostram que a produtividade de grãos está correlacionada com o número de vagens por planta, número de grãos por planta e massa de grãos, que são, portanto, variáveis importantes na seleção de genótipos produtivos. A variação dos componentes da produção do feijoeiro colabora com a manutenção da estabilidade da produtividade de grãos, ou seja, no caso de um desses componentes ser prejudicado por qualquer fator, outro componente se eleva, estabilizando a produtividade (Costa et al., 1983; Coimbra et al., 1999).

No ano de 2001, a temperatura média foi mais favorável ao desenvolvimento do feijoeiro, ficando em torno de $22^{\circ} \mathrm{C}$ ao longo do ciclo (Figura 1). Situação diferente ocorreu no ano de 2002, quando a temperatura média oscilou bastante. Com aproximadamente $15 \mathrm{DAS}$, verificou-se temperatura média próxima a $10^{\circ} \mathrm{C}$ e, a partir de 48 DAS, observou-se temperatura média acima de $30^{\circ} \mathrm{C}$ aproximando-se de $40^{\circ} \mathrm{C}$ aos 57 e $72 \mathrm{DAS}$, coincidindo com as fases reprodutivas do florescimento pleno, início da formação das vagens e enchimento das vagens. Essas condições interferiram no número de grãos por vagens que foi de 3,3 na média, sendo muito inferior ao obtido no ano anterior (4,5 grãos por vagem), concordando com os resultados de Didonet (2002), que ve- 
rificou o efeito maléfico de altas temperaturas nesse componente da produção.

Houve diferença significativa entre os genótipos de feijão em relação ao teor de proteína bruta e o tempo de cozimento nos dois anos (Tabela 3 ).

O teor de proteína bruta variou de $18,6 \%$ (Rudá) a 23,8\% (Porto Real e EL 49) e de 17,0\% (CNFC 8007 e CNFC 8011) a 21,8\% (EL 49) nos anos de 2001 e 2002, respectivamente. Entre os genótipos avaliados, apenas Campeão 2, Rudá e IAPAR 81 não apresentaram teor de proteína bruta acima de $20 \%$ no ano de 2001. Já em 2002, apenas os genótipos Carioca, CNFC 8005 e EL 49 apresentaram teor de proteína acima de 20,0\%. Esses mesmos genótipos apresentaram teor de proteína bruta médio superior a esse valor nos dois anos.

Os teores protéicos dos grãos foram semelhantes aos obtidos por Párraga et al. (1981) e inferiores aos obtidos por Pimentel et al. (1988). Segundo Lajolo et al. (1996), na composição centesimal do feijão, o conteúdo protéico é variável em razão do local de cultivo, de fatores ambientais e da própria cultivar. No entanto, os genótipos que apresentaram os maiores teores de proteína nem sempre sobressaíram-se nas outras características avaliadas, especialmente em relação à produtividade de grãos, tais como os genótipos Porto Real e EL 49, no ano de 2001. Já os genótipos IAC-Carioca e CNFC 8010 proporcionaram as maiores produtividades em 2001, mas o teor de proteína bruta foi igual entre si e abaixo da média experimental. Em 2002, os genótipos CNFC 8005 e EL 49 proporcionaram teores de proteína bruta acima de $21,0 \%$ e produtividade de grãos abaixo da média experimental. Esses resultados corroboram os dados de Bressani (1989) e Pompeu (1993), ou seja, o teor de proteína bruta é inversamente proporcional à produtividade de grãos.

Quanto ao tempo de cozimento, destacaram-se com valores inferiores a 20 minutos, nos dois anos, os genótipos Carioca, IAC-Carioca Eté, Princesa, IAPAR 14, IAPAR 80, Porto Real, CNFC 8006, CNFC 8013 e MA 534534. Já o genótipo CNFC 8005 obteve o maior tempo de cozimento nos dois anos, de 25 e 23 minutos, respectivamente. No entanto, o tempo de

Tabela 1. Produtividade de grãos e índices relativos em relação à cultivar Carioca (IRC) e à cultivar Pérola (IRP) de genótipos de feijão, cultivados na época das águas ${ }^{(1)}$.

\begin{tabular}{|c|c|c|c|c|c|c|}
\hline \multirow[t]{2}{*}{ Genótipos } & \multicolumn{2}{|c|}{ Produtividade $\left(\mathrm{kg} \mathrm{ha}^{-1}\right)$} & \multicolumn{2}{|c|}{ IRC $(\%)$} & \multicolumn{2}{|c|}{ IRP $(\%)$} \\
\hline & 2001 & 2002 & 2001 & 2002 & 2001 & 2002 \\
\hline Carioca & $2.462 \mathrm{hijk}$ & $2.940 \mathrm{abc}$ & 100 & 100 & 93 & 155 \\
\hline Pérola & 2.661 efghi & $1.900 \mathrm{ghij}$ & 108 & 65 & 100 & 100 \\
\hline IAC-Carioca & $3.555 \mathrm{ab}$ & $2.511 \mathrm{bcdefg}$ & 144 & 85 & 134 & 132 \\
\hline IAC-Carioca Eté & $2.363 \mathrm{ijk}$ & $1.596 \mathrm{ij}$ & 96 & 54 & 89 & 84 \\
\hline IAC-Carioca Pyatã & $2.655 \mathrm{efghi}$ & $1.449 \mathrm{j}$ & 108 & 49 & 100 & 76 \\
\hline IAC-Carioca Aruã & $2.237 \mathrm{jk}$ & $2.046 \mathrm{fghij}$ & 91 & 70 & 84 & 108 \\
\hline Campeão 2 & $2.581 \mathrm{fghij}$ & 2.130defghi & 105 & 72 & 97 & 112 \\
\hline Carioca Precoce & $2.151 \mathrm{k}$ & $1.878 \mathrm{hij}$ & 87 & 64 & 81 & 99 \\
\hline FT-Bonito & $2.862 \mathrm{cdefgh}$ & $2.701 \mathrm{bcde}$ & 116 & 92 & 108 & 142 \\
\hline Rudá & $3.029 \mathrm{cde}$ & $2.714 \mathrm{bcde}$ & 123 & 92 & 114 & 143 \\
\hline Aporé & $2.545 \mathrm{ghijk}$ & 2.122efghi & 103 & 72 & 96 & 112 \\
\hline Princesa & $2.895 \mathrm{cdefg}$ & 2.000fghij & 118 & 68 & 109 & 105 \\
\hline IAPAR 14 & 2.689defghi & 2.199defghi & 109 & 75 & 101 & 116 \\
\hline IAPAR 80 & $3.132 \mathrm{c}$ & $2.090 \mathrm{efghi}$ & 127 & 71 & 118 & 110 \\
\hline IAPAR 81 & $3.114 \mathrm{c}$ & 2.178 defghi & 126 & 74 & 117 & 115 \\
\hline Porto Real & $2.946 \mathrm{cdefg}$ & $2.896 \mathrm{abc}$ & 120 & 99 & 111 & 152 \\
\hline CNFC 8005 & $2.947 \mathrm{cdefg}$ & $1.449 \mathrm{j}$ & 120 & 49 & 111 & 76 \\
\hline CNFC 8006 & 2.931cdefg & $2.155 \mathrm{defghi}$ & 119 & 73 & 110 & 113 \\
\hline CNFC 8007 & $3.183 \mathrm{bc}$ & $2.222 \mathrm{defghi}$ & 129 & 76 & 120 & 117 \\
\hline CNFC 8008 & $3.042 \mathrm{cde}$ & $2.754 \mathrm{bcd}$ & 124 & 94 & 114 & 145 \\
\hline CNFC 8009 & $2.237 \mathrm{jk}$ & $2.946 \mathrm{abc}$ & 91 & 100 & 84 & 155 \\
\hline CNFC 8010 & $3.623 \mathrm{a}$ & $2.029 \mathrm{fghij}$ & 147 & 69 & 136 & 107 \\
\hline CNFC 8011 & $3.000 \mathrm{cde}$ & $2.585 \mathrm{bcdef}$ & 122 & 88 & 113 & 136 \\
\hline CNFC 8012 & $3.086 \mathrm{~cd}$ & $3.412 \mathrm{a}$ & 125 & 116 & 116 & 180 \\
\hline CNFC 8013 & $2.977 \mathrm{cdef}$ & $2.364 \mathrm{cdefgh}$ & 121 & 80 & 112 & 124 \\
\hline CNFC 8156 & $3.192 b c$ & $3.073 \mathrm{ab}$ & 130 & 105 & 120 & 162 \\
\hline RELAV 37-19 & $2.920 \mathrm{cdefg}$ & 2.138defghi & 119 & 73 & 110 & 113 \\
\hline MA 534534 & 2.691 defghi & $2.598 \mathrm{bcdef}$ & 109 & 88 & 101 & 137 \\
\hline EL 49 & $2.464 \mathrm{hijk}$ & $2.144 \mathrm{defghi}$ & 100 & 73 & 93 & 113 \\
\hline
\end{tabular}

${ }^{(1)}$ Médias seguidas de pelo menos uma mesma letra, na vertical, não diferem entre si pelo teste de Tukey a 5\% de probabilidade; os coeficientes de variação em relação à produtividade de grãos foram de 5,3\% e 9,9\%, respectivamente, nos anos 2001 e 2002. 
cozimento dos diferentes genótipos variou de 15 a $25 \mathrm{mi}-$ nutos e de 15 a 23 minutos em relação aos anos de 2001 e 2002, respectivamente, com variação de 10 e 8 minutos.

O tempo de cozimento do feijão é influenciado pela cultivar (Durigan et al., 1978; Pimentel et al., 1988; Vieira et al., 1989; Cazetta et al., 1995), tempo transcorrido após a colheita e histórico do armazenamento (Chiaradia \& Gomes, 1997; Ramos Júnior, 2002), além das condições ambientais (Bressani, 1989; Lam-Sanchez et al., 1990), do sistema e da temperatura utilizados no processo de cocção.

No ano de 2001, em apenas sete genótipos houve a presença de grãos de casca dura ("hardshell”) sem capacidade de absorver água, cuja porcentagem variou de 0,1 a $0,5 \%$. Em 2002, 15 materiais apresentaram casca dura que variou de $1,0 \%$ a $1,6 \%$. Esses valores são considerados baixos ou de reduzida intensidade, mostrando de forma geral, que o grupo de genótipos apresentou comportamento favorável quanto a essa característica. Moreno \& Lopez (1992) relataram que, para prevenir a ocorrência de casca dura, indica-se o armazenamento sob temperaturas inferiores a $17^{\circ} \mathrm{C}$, umidade relativa abaixo de $55 \%$ e umidade de grãos em torno de $8 \%$.

As equações de regressão entre o tempo de hidratação e a quantidade de água absorvida para os genótipos de feijão evidenciaram que o período de máxima hidratação variou de 8 horas e 25 minutos a 15 horas e 44 minutos e de 8 horas e 8 minutos a 14 horas e 28 minutos para os anos de 2001 e 2002 , respectivamente, tendo portanto variação de 7 horas e 19 minutos e 6 horas e 20 minutos, considerados elevados (Tabelas 4 e 5). No entanto, todos os genótipos, até mesmo aqueles que necessitaram de mais tempo para atingir a máxima hidratação, com exceção da cultivar IAC-Carioca Pyatã, podem ser considerados de comportamento satisfatório. Assim, Durigan et al. (1978) observaram que não há relação entre capacidade de hidratação com o tempo de cozimento, sugerindo que a mesma cultivar de feijão pode apresentar péssimas características de hidratação, mas ótimo comportamento quanto ao seu cozimento, ou vice-versa. No entanto,

Tabela 2. Número de vagens por planta, número de grãos por vagem e massa de 100 grãos de genótipos de feijão, cultivados na época das águas ${ }^{(1)}$.

\begin{tabular}{|c|c|c|c|c|c|c|}
\hline \multirow[t]{2}{*}{ Genótipos } & \multicolumn{2}{|c|}{ Vagens/planta } & \multicolumn{2}{|c|}{ Grãos/vagem } & \multicolumn{2}{|c|}{ Massa de 100 grãos (\%) } \\
\hline & 2001 & 2002 & 2001 & 2002 & 2001 & 2002 \\
\hline Carioca & $11,6 a b c d$ & $16,3 \mathrm{bcd}$ & 4,8 & $3,5 \mathrm{ab}$ & $26,2 \mathrm{ab}$ & 28,5bcdefghi \\
\hline Pérola & $12,2 \mathrm{abcd}$ & $9,3 b c$ & 3,8 & $2,5 \mathrm{ab}$ & $30,4 \mathrm{ab}$ & $35,8 \mathrm{a}$ \\
\hline IAC-Carioca & $13,3 \mathrm{abcd}$ & $17,5 \mathrm{bcd}$ & 4,5 & $3,0 \mathrm{ab}$ & $23,8 b$ & $30,0 \mathrm{bcdef}$ \\
\hline IAC-Carioca Eté & $11,3 \mathrm{bcd}$ & $15,3 \mathrm{~cd}$ & 3,8 & $2,8 \mathrm{ab}$ & $23,7 b$ & $23,5 \mathrm{k}$ \\
\hline IAC-Carioca Pyatã & $10,8 \mathrm{bcd}$ & $20,3 \mathrm{abc}$ & 4,0 & $1,8 \mathrm{~b}$ & $23,6 b$ & $24,5 \mathrm{ijk}$ \\
\hline IAC-Carioca Aruã & $12,8 \mathrm{abcd}$ & $18,5 \mathrm{bcd}$ & 4,0 & $3,0 \mathrm{ab}$ & $21,8 \mathrm{~b}$ & 26,3 efghijk \\
\hline Campeão 2 & $14,3 \mathrm{abcd}$ & $16,8 \mathrm{bcd}$ & 4,5 & $2,8 \mathrm{ab}$ & $27,0 \mathrm{ab}$ & $31,0 \mathrm{bcd}$ \\
\hline Carioca Precoce & $13,7 \mathrm{abcd}$ & $16,3 \mathrm{bcd}$ & 4,8 & $3,5 \mathrm{ab}$ & $25,3 \mathrm{ab}$ & $25,5 \mathrm{ghijk}$ \\
\hline FT-Bonito & $10,4 \mathrm{~cd}$ & $19,5 \mathrm{bc}$ & 4,8 & $3,3 \mathrm{ab}$ & $26,2 \mathrm{ab}$ & 30,5 bcde \\
\hline Rudá & $15,0 \mathrm{abcd}$ & $20,5 \mathrm{abc}$ & 4,0 & $3,5 \mathrm{ab}$ & $21,6 b$ & 27,0defghilk \\
\hline Aporé & $12,1 \mathrm{abcd}$ & $13,8 \mathrm{~cd}$ & 4,3 & $3,3 \mathrm{ab}$ & $28,6 \mathrm{ab}$ & $32,3 \mathrm{ab}$ \\
\hline Princesa & $17,5 \mathrm{a}$ & $14,8 \mathrm{~cd}$ & 4,5 & $4,0 \mathrm{a}$ & $23,1 b$ & $24,8 \mathrm{ijk}$ \\
\hline IAPAR 14 & $14,5 \mathrm{abcd}$ & $19,0 \mathrm{bc}$ & 4,0 & $3,0 \mathrm{ab}$ & $24,6 b$ & 25,8 fghijk \\
\hline IAPAR 80 & $14,8 \mathrm{abcd}$ & $17,8 \mathrm{bcd}$ & 4,5 & $2,8 \mathrm{ab}$ & $23,8 \mathrm{~b}$ & 27,8 cdefghijk \\
\hline IAPAR 81 & $11,9 \mathrm{abcd}$ & $16,8 \mathrm{bcd}$ & 4,5 & $3,5 \mathrm{ab}$ & $25,7 \mathrm{ab}$ & 29,8 bcdefg \\
\hline Porto Real & $15,8 \mathrm{abc}$ & $24,3 \mathrm{ab}$ & 4,8 & $3,5 \mathrm{ab}$ & $25,6 \mathrm{ab}$ & 29,3 bcdefgh \\
\hline CNFC 8005 & $12,6 a b c d$ & $10,0 \mathrm{~d}$ & 4,3 & $2,8 \mathrm{ab}$ & $29,6 \mathrm{ab}$ & 29,5 bcdefgh \\
\hline CNFC 8006 & $14,1 \mathrm{abcd}$ & $21,3 \mathrm{abc}$ & 4,3 & $3,5 \mathrm{ab}$ & $24,0 \mathrm{~b}$ & $24,8 \mathrm{ijk}$ \\
\hline CNFC 8007 & $12,7 \mathrm{abcd}$ & $13,5 \mathrm{~cd}$ & 5,0 & $4,0 \mathrm{a}$ & $23,2 b$ & 26,3 efghijk \\
\hline CNFC 8008 & $11,2 \mathrm{bcd}$ & $16,8 \mathrm{bcd}$ & 5,0 & $3,8 \mathrm{a}$ & $27,4 \mathrm{ab}$ & $31,0 \mathrm{bcd}$ \\
\hline CNFC 8009 & $16,0 \mathrm{ab}$ & $15,0 \mathrm{~cd}$ & 4,0 & $3,8 \mathrm{a}$ & $35,5 \mathrm{a}$ & 29,3 bcdefgh \\
\hline CNFC 8010 & $11,7 \mathrm{abcd}$ & $14,3 \mathrm{~cd}$ & 5,0 & $3,5 \mathrm{ab}$ & $24,6 b$ & $25,3 \mathrm{hijk}$ \\
\hline CNFC 8011 & $12,6 \mathrm{abcd}$ & $17,8 \mathrm{bcd}$ & 5,0 & $4,0 \mathrm{a}$ & $24,2 \mathrm{~b}$ & $24,8 \mathrm{ijk}$ \\
\hline CNFC 8012 & $10,7 \mathrm{bcd}$ & $13,5 \mathrm{~cd}$ & 5,3 & $3,5 \mathrm{ab}$ & $25,9 \mathrm{ab}$ & 28,0 bcdefghij \\
\hline CNFC 8013 & $11,2 \mathrm{bcd}$ & $16,8 \mathrm{bcd}$ & 4,3 & $3,0 \mathrm{ab}$ & $21,7 b$ & $24,0 \mathrm{jk}$ \\
\hline CNFC 8156 & $12,3 \mathrm{abcd}$ & $13,8 \mathrm{~cd}$ & 4,8 & $3,8 \mathrm{a}$ & $24,0 \mathrm{~b}$ & 27,5 defghijk \\
\hline RELAV 37-19 & $12,6 a b c d$ & $16,5 \mathrm{bcd}$ & 4,8 & $4,0 \mathrm{a}$ & $25,1 \mathrm{ab}$ & 27,8 cdefghijk \\
\hline MA 534534 & 13,4 abcd & $28,5 \mathrm{a}$ & 3,8 & $3,0 \mathrm{ab}$ & $25,0 \mathrm{ab}$ & 28,5 bcdefghi \\
\hline EL 49 & $9,4 \mathrm{~d}$ & $16,0 \mathrm{bcd}$ & 4,5 & $3,0 \mathrm{ab}$ & $30,1 \mathrm{ab}$ & $32,0 \mathrm{abc}$ \\
\hline $\mathrm{CV}(\%)$ & 16,83 & 18,5 & 14,17 & 20,96 & 15,21 & 5,64 \\
\hline
\end{tabular}

${ }^{11}$ Médias seguidas de pelo menos uma mesma letra, na vertical, não diferem entre si pelo teste de Tukey a 5\% de probabilidade. 
Tabela 3. Teor de proteína bruta, tempo para cozimento e grãos de casca dura que não reidrataram em genótipos de feijão, cultivados na época das águas ${ }^{(1)}$.

\begin{tabular}{|c|c|c|c|c|c|c|}
\hline \multirow[t]{2}{*}{ Genótipos } & \multicolumn{2}{|c|}{ Teor de proteína bruta $(\%)$} & \multicolumn{2}{|c|}{ Tempo para cozimento $(\mathrm{min})$} & \multicolumn{2}{|c|}{ Grãos de casca dura $(\%)$} \\
\hline & 2001 & 2002 & 2001 & 2002 & 2001 & 2002 \\
\hline Carioca & $21,5 \mathrm{ab}$ & $21,0 \mathrm{abc}$ & $15 \mathrm{f}$ & $16 \mathrm{fg}$ & 0,0 & 0,1 \\
\hline Pérola & $21,9 \mathrm{ab}$ & 19,0 abcd & $20 \mathrm{bcde}$ & 21 abcd & 0,0 & 0,4 \\
\hline IAC-Carioca & $21,6 \mathrm{ab}$ & $18,5 \mathrm{abcd}$ & 20 bcde & $21 \mathrm{abcd}$ & 0,4 & 0,0 \\
\hline IAC-Carioca Eté & $21,1 \mathrm{ab}$ & 19,5 abcd & $17 \mathrm{~d}$ & $16 \mathrm{efg}$ & 0,0 & 0,3 \\
\hline IAC-Carioca Pyatã & $22,2 \mathrm{ab}$ & $18,5 \mathrm{abcd}$ & $20 \mathrm{bcde}$ & $21 \mathrm{abcd}$ & 0,1 & 1,3 \\
\hline IAC-Carioca Aruã & $22,2 \mathrm{ab}$ & $17,5 \mathrm{~d}$ & 21 abcd & $19 \mathrm{bcdef}$ & 0,0 & 0,0 \\
\hline Campeão 2 & $19,4 \mathrm{ab}$ & $17,3 \mathrm{~cd}$ & $21 \mathrm{abcd}$ & 21 abcd & 0,0 & 0,1 \\
\hline Carioca Precoce & $22,4 \mathrm{ab}$ & $18,8 \mathrm{abcd}$ & $17 \mathrm{~d}$ & 21 abcd & 0,2 & 0,3 \\
\hline FT-Bonito & $21,2 \mathrm{ab}$ & $18,3 \mathrm{abcd}$ & $19 b c d e f$ & 20 abcd & 0,0 & 0,0 \\
\hline Rudá & $18,6 b$ & $17,3 \mathrm{~cd}$ & $20 \mathrm{bcde}$ & 20 abcd & 0,0 & 0,3 \\
\hline Aporé & $23,4 \mathrm{ab}$ & $18,8 \mathrm{abcd}$ & $23 a b$ & $22 a b c$ & 0,0 & 0,0 \\
\hline Princesa & $22,0 \mathrm{ab}$ & $17,5 \mathrm{~cd}$ & $18 \mathrm{cdef}$ & 19 bcdef & 0,0 & 0,4 \\
\hline IAPAR 14 & $21,8 \mathrm{ab}$ & $17,3 \mathrm{~cd}$ & $18 \mathrm{cdef}$ & $19 \mathrm{bcdef}$ & 0,0 & 0,1 \\
\hline IAPAR 80 & $20,7 \mathrm{ab}$ & $18,5 \mathrm{abcd}$ & $15 \mathrm{f}$ & $15 \mathrm{~g}$ & 0,0 & 0,0 \\
\hline IAPAR 81 & $19,5 \mathrm{ab}$ & $17,8 \mathrm{bcd}$ & 20 bcde & 21 abcd & 0,0 & 0,5 \\
\hline Porto Real & $23,8 \mathrm{a}$ & 18,0 abcd & $16 \mathrm{ef}$ & 18 defg & 0,0 & 0,3 \\
\hline CNFC 8005 & $22,3 \mathrm{ab}$ & $21,5 \mathrm{ab}$ & $25 a$ & $23 a$ & 0,5 & 0,0 \\
\hline CNFC 8006 & $22,1 \mathrm{ab}$ & 18,0 abcd & $19 \mathrm{bcdef}$ & $18 \mathrm{cdefg}$ & 0,0 & 0,5 \\
\hline CNFC 8007 & $20,5 \mathrm{ab}$ & $17,0 \mathrm{~d}$ & $20 \mathrm{bcde}$ & $20 \mathrm{abcd}$ & 0,0 & 0,0 \\
\hline CNFC 8008 & $22,7 \mathrm{ab}$ & $17,3 \mathrm{~cd}$ & 21 abcd & $21 \mathrm{abcd}$ & 0,4 & 0,3 \\
\hline CNFC 8009 & $22,7 \mathrm{ab}$ & $19,0 \mathrm{abcd}$ & $23 \mathrm{ab}$ & $21 \mathrm{abcd}$ & 0,0 & 0,0 \\
\hline CNFC 8010 & $20,3 \mathrm{ab}$ & $19,3 \mathrm{abcd}$ & 20 bcde & $21 \mathrm{abcd}$ & 0,4 & 1,6 \\
\hline CNFC 8011 & $20,0 \mathrm{ab}$ & $17,0 \mathrm{~d}$ & 21 abcd & 21 abcd & 0,0 & 0,0 \\
\hline CNFC 8012 & $22,3 \mathrm{ab}$ & $19,5 \mathrm{abcd}$ & $20 \mathrm{bcde}$ & 20 abcd & 0,0 & 0,0 \\
\hline CNFC 8013 & $21,6 a b$ & $17,8 \mathrm{bcd}$ & $17 \mathrm{def}$ & 18 bcdefg & 0,0 & 0,0 \\
\hline CNFC 8156 & $21,9 \mathrm{ab}$ & $18,5 \mathrm{abcd}$ & $20 \mathrm{bcde}$ & 20abcd & 0,0 & 0,0 \\
\hline RELAV 37-19 & $23,2 \mathrm{ab}$ & $19,5 \mathrm{abcd}$ & $22 \mathrm{abc}$ & $22 \mathrm{abc}$ & 0,5 & 0,2 \\
\hline MA 534534 & $20,7 \mathrm{ab}$ & $18,5 \mathrm{abcd}$ & $18 \mathrm{cdef}$ & 19 bcdef & 0,0 & 0,0 \\
\hline EL 49 & $23,8 \mathrm{a}$ & $21,8 \mathrm{a}$ & $22 a b c$ & $22 \mathrm{abc}$ & 0,0 & 0,0 \\
\hline $\mathrm{CV}(\%)$ & 7,4 & 7,8 & 8,86 & 6,99 & - & - \\
\hline
\end{tabular}

Tabela 4. Regressão entre o tempo para hidratação e a quantidade de água absorvida pelos grãos de genótipos de feijão, cultivados na época das águas no ano de 2001.

\begin{tabular}{|c|c|c|c|}
\hline Genótipos & Equação de regressão $^{(1)}$ & $\mathrm{R}^{2}$ & Tempo de máxima hidratação (horas:minutos) \\
\hline Carioca & $Y=10,2175+7,5976 X-0,4334 X^{2}$ & 0,8939 & $8: 46$ \\
\hline Pérola & $Y=7,5852+8,1180 X-0,4312 X^{2}$ & 0,9535 & $9: 25$ \\
\hline IAC-Carioca & $Y=6,0461+7,4547 X-0,3512 X^{2}$ & 0,9809 & $10: 37$ \\
\hline IAC-Carioca Eté & $Y=2,7006+7,3917 X-0,3466 X^{2}$ & 0,9902 & $10: 40$ \\
\hline IAC-Carioca Pyatã & $\mathrm{Y}=0,1122+5,8059 \mathrm{X}-0,1844 \mathrm{X}^{2}$ & 0,9928 & $15: 44$ \\
\hline IAC-Carioca Aruã & $Y=13,8334+7,4688 X-0,4311 X^{2}$ & 0,8438 & $8: 40$ \\
\hline Campeão 2 & $Y=9,9154+8,2297 X-0,4755 X^{2}$ & 0,9078 & $8: 39$ \\
\hline Carioca Precoce & $Y=8,2496+8,1508 X-0,4638 X^{2}$ & 0,9311 & $8: 47$ \\
\hline FT-Bonito & $Y=8,4378+7,7648 X-0,4079 X^{2}$ & 0,9382 & $9: 31$ \\
\hline Rudá & $Y=8,4761+8,2560 X-0,4637 X^{2}$ & 0,9261 & $8: 54$ \\
\hline Aporé & $Y=8,5102+8,7954 X-0,5176 X^{2}$ & 0,9337 & $8: 30$ \\
\hline Princesa & $Y=7,0512+7,3855 X-0,3562 X^{2}$ & 0,9714 & $10: 22$ \\
\hline IAPAR 14 & $\mathrm{Y}=4,4432+8,0354 \mathrm{X}-0,4010 \mathrm{X}^{2}$ & 0,9857 & 10:01 \\
\hline IAPAR 80 & $Y=11,4165+8,0190 X-0,4627 X^{2}$ & 0,9009 & $8: 40$ \\
\hline IAPAR 81 & $Y=9,2456+8,6070 X-0,5114 X^{2}$ & 0,9180 & $8: 25$ \\
\hline Porto Real & $Y=8,1153+7,3664 X-0,3707 X^{2}$ & 0,9601 & $9: 56$ \\
\hline CNFC 8005 & $Y=9,3331+8,2901 X-0,4678 X^{2}$ & 0,9276 & $8: 52$ \\
\hline CNFC 8006 & $Y=8,2499+7,8711 X-0,4291 X^{2}$ & 0,9522 & $9: 10$ \\
\hline CNFC 8007 & $Y=4,6526+8,1678 X-0,4322 X^{2}$ & 0,9770 & $9: 27$ \\
\hline CNFC 8008 & $Y=8,9476+8,0756 X-0,4577 X^{2}$ & 0,9219 & $8: 49$ \\
\hline CNFC 8009 & $Y=6,5305+8,8070 X-0,5055 X^{2}$ & 0,9530 & $8: 43$ \\
\hline CNFC 8010 & $\mathrm{Y}=4,2506+7,1035 \mathrm{X}-0,3322 \mathrm{X}^{2}$ & 0,9843 & $10: 41$ \\
\hline CNFC 8011 & $Y=11,6785+7,9690 X-0,4555 X^{2}$ & 0,8921 & $8: 45$ \\
\hline CNFC 8012 & $Y=3,6308+7,7142 X-0,3814 X^{2}$ & 0,9866 & 10:07 \\
\hline CNFC 8013 & $Y=12,1230+7,3405 X-0,4130 X^{2}$ & 0,8748 & $8: 53$ \\
\hline CNFC 8156 & $Y=7,5689+8,9908 X-0,5125 X^{2}$ & 0,9573 & $8: 46$ \\
\hline RELAV 37-19 & $Y=5,8485+8,3882 X-0,4420 X^{2}$ & 0,9718 & $9: 29$ \\
\hline MA 534534 & $Y=11,7146+7,8681 X-0,4517 X^{2}$ & 0.8869 & $8: 43$ \\
\hline EL 49 & $Y=3,2527+7,4443 X-0,3487 X^{2}$ & 0,9913 & $10: 40$ \\
\hline
\end{tabular}

${ }^{(1)} \mathrm{X}$ : tempo para hidratação (horas); Y: quantidade de água absorvida (mL). 
Tabela 5. Regressão entre o tempo para hidratação e a quantidade de água absorvida pelos grãos de genótipos de feijão, cultivados na época das águas no ano de 2002.

\begin{tabular}{|c|c|c|c|}
\hline Genótipos & Equação de regressão $^{(1)}$ & $\mathrm{R}^{2}$ & Tempo de máxima hidratação (horas:minutos) \\
\hline Carioca & $\mathrm{Y}=8,8681+8,2250 \mathrm{X}-0,4869 \mathrm{X}^{2}$ & 0,9307 & $8: 26$ \\
\hline Pérola & $Y=6,1708+8,0215 X-0,4185 X^{2}$ & 0,9726 & $9: 35$ \\
\hline IAC-Carioca & $Y=6,5353+8,5350 X-0,4557 X^{2}$ & 0,9623 & $9: 22$ \\
\hline IAC-Carioca Eté & $Y=3,5115+7,5255 X-0,3719 X^{2}$ & 0,9895 & $10: 07$ \\
\hline IAC-Carioca Pyatã & $Y=2,2345+5,7241 X-0,1979 X^{2}$ & 0,9860 & $14: 28$ \\
\hline IAC-Carioca Aruã & $Y=16,5780+7,4689 X-0,4595 X^{2}$ & 0,7688 & 8:08 \\
\hline Campeão 2 & $Y=8,8021+7,7306 X-0,4263 X^{2}$ & 0,9330 & 9:08 \\
\hline Carioca Precoce & $Y=6,8211+7,0480 X-0,3602 X^{2}$ & 0,9550 & $9: 47$ \\
\hline FT-Bonito & $Y=12,2326+7,6905 X-0,4383 X^{2}$ & 0,8764 & $8: 47$ \\
\hline Rudá & $Y=7,9306+7,5455 X-0,4044 X^{2}$ & 0,9484 & $9: 20$ \\
\hline Aporé & $Y=7,7070+8,5326 X-0,4761 X^{2}$ & 0,9279 & $8: 46$ \\
\hline Princesa & $Y=6,1290+6,7975-0,3198 X^{2}$ & 0,9597 & $10: 38$ \\
\hline IAPAR 14 & $Y=5,2147+8,5991 X-0,5010 X^{2}$ & 0,9566 & $8: 35$ \\
\hline IAPAR 80 & $Y=15,5380+6,3162 X-0,3519 X^{2}$ & 0,7846 & $8: 59$ \\
\hline IAPAR 81 & $Y=9,7349+7,5362 X-0,4233 X^{2}$ & 0,9157 & $8: 54$ \\
\hline Porto Real & $Y=5,3507+6,8229 X-0,3241 X^{2}$ & 0,9772 & $8: 41$ \\
\hline CNFC 8005 & $Y=10,3097+8,0218 X-0,4619 X^{2}$ & 0,9058 & $10: 32$ \\
\hline CNFC 8006 & $Y=8,4725+6,6725 X-0,3336 X^{2}$ & 0,9360 & 10:00 \\
\hline CNFC 8007 & $Y=13,4833+7,9464 X-0,4800 X^{2}$ & 0,8342 & $8: 17$ \\
\hline CNFC 8008 & $Y=10,7731+8,4658 X-0,5053 X^{2}$ & 0,9017 & $8: 23$ \\
\hline CNFC 8009 & $Y=10,1810+7,7535 X-0,4381 X^{2}$ & 0,9051 & $8: 51$ \\
\hline CNFC 8010 & $Y=6,1798+7,8583 X-0,4276 X^{2}$ & 0,9562 & $9: 41$ \\
\hline CNFC 8011 & $Y=6,8835+7,8583 X-0,4276 X^{2}$ & 0,9537 & $9: 11$ \\
\hline CNFC 8012 & $Y=2,6410+6,8444 X-0,3121 X^{2}$ & 0,9900 & $10: 58$ \\
\hline CNFC 8013 & $Y=9,6371+7,1584 X-0,3922 X^{2}$ & 0,9196 & 9:08 \\
\hline CNFC 8156 & $Y=10,4024+7,7044 X-0,4221 X^{2}$ & 0,9098 & 9:08 \\
\hline RELAV 37-19 & $Y=10,4147+7,1923 X-0,3938 X^{2}$ & 0,8920 & 9:08 \\
\hline MA 534534 & $Y=9,4905+7,6508 X-0,4213 X^{2}$ & 0,9194 & 9:05 \\
\hline EL 49 & $\mathrm{Y}=9,3078+7,9279 \mathrm{X}-0,4416 \mathrm{X}^{2}$ & 0,9215 & $8: 59$ \\
\hline
\end{tabular}

${ }^{(1)} \mathrm{X}$ : tempo para hidratação (horas); Y: quantidade de água absorvida (mL).

Lam-Sanchez et al. (1990) concluíram que diferentes épocas de semeadura afetaram as características de hidratação e o tempo de cozimento dos grãos de cultivares de feijão. Na época da seca, verificaram altos valores de grãos com casca dura nas cultivares Ayso e Carioca 80, 19,5\% e 7,4\%, respectivamente. Já na época das águas, a porcentagem destes grãos foi quase nula, a não ser na cultivar Paraná-1 que apresentou 4,5\%.

\section{Conclusões}

1. Os genótipos IAC-Carioca, FT-Bonito, Rudá, Porto Real, CNFC 8008, CNFC 8011, CNFC 8012, CNFC 8013 e CNFC 8156 são os mais produtivos.

2. Os genótipos IAC-Carioca, CNFC 8012 e CNFC 8156 apresentam alta produtividade e reduzido tempo para cozimento.

\section{Agradecimentos}

À Embrapa Arroz e Feijão, pela cessão das sementes dos genótipos; à Fapesp pelas bolsas concedidas aos autores Ricardo Soares de Oliveira e Tiago Roque Benetoli da Silva.

\section{Referências}

BRESSANI, R. Revision sobre la calidad del grano de frijol. Archivos Latinoamericanos de Nutricion, v.39, p.419-442, 1989.

CARBONELL, S.A.M.; AZEVEDO FILHO, J.A.; DIAS, L.A.S.; GONÇALVES, C.; ANTONIO, C.B. Adaptabilidade e estabilidade de produção de cultivares e linhagens de feijoeiro no Estado de São Paulo. Bragantia, v.60, p.69-77, 2001.

CARBONELL, S.A.M.; ITO, M.F.; AZEVEDO FILHO, J.A. de; SARTORI, J.A. Cultivares comerciais de feijoeiro para o Estado de São Paulo: características e melhoramento. In: DIA DE CAMPO DE FEIJÃO, 19., 2003, Capão Bonito. Anais. Campinas: Instituto Agronômico, 2003. p.5-27. (Documentos IAC, 71).

CARBONELL, S.A.M.; POMPEU, A.S. Estabilidade fenotípica de linhagens de feijoeiro em três épocas de plantio no Estado de São Paulo. Pesquisa Agropecuária Brasileira, v.35, p.321-329, 2000.

CAZETTA, J.O.; KANESHIRO, M.A.B.; FALEIROS, R.R.S.; DURIGAN, J.F. Comparação de aspectos químicos e tecnológicos de grãos verdes e maduros de guandu com os de feijão e ervilha. Alimentos e Nutrição, v.6, p.39-53, 1995.

CHIARADIA, A.C.N.; GOMES, J.C. Feijão: química, nutrição e tecnologia. Viçosa: UFV, 1997. 180p.

COIMBRA, J.L.M.; GUIDOLIN, A.F.; CARVALHO, F.I.F.; COIMBRA, S.M.M.; MARCHIORO, V.S. Análise de trilha - I: análise do rendimento de grãos e seus componentes. Ciência Rural, v.29, p.213-218, 1999. 
COSTA, J.G.C.; KOHASHI-SHIBATA, J.; COLIN, S.M Plasticidade no feijoeiro comum. Pesquisa Agropecuária Brasileira, v.18, p.159-167, 1983.

DIDONET, A.D. Respostas da cultivar de feijoeiro comum Pérola ao choque térmico com altas temperaturas. Santo Antônio de Goiás: Embrapa-CNPAF, 2002. 3p. (Comunicado Técnico, 39).

DUARTE, J.B.; ZIMMERMANN, M.J.O. Adaptabilidade e estabilidade de rendimento de genótipos de feijoeiro comum. Pesquisa Agropecuária Brasileira, v.29, p.25-32, 1994.

DURIGAN, J.F. Influência do tempo e das condições de estocagem sobre as propriedades químicas, físico-mecânicas e nutricionais do feijão mulatinho (Phaseolus vulgaris L.). Campinas, 1979. 81p. Dissertação (Mestrado) - Universidade de Campinas, Campinas.

DURIGAN, J.F.; FALEIROS, R.R.S.; LAM-SANCHEZ, A. Determinação das características tecnológicas e nutricionais de diversas variedades de feijão (Phaseolus vulgaris L.) - I: características tecnológicas. Científica, v.6, p.215-224, 1978.

FERREIRA, C.M.; DEL PELOSO, M.J.; FARIA, L.C. Feijão na economia nacional. Santo Antônio de Goiás: Embrapa-CNPAF, 2002. 47p. (Documentos, 135).

IADEROZA, M.; SALES, A.M.; BALDINI, V.L.S.; SARTORI, M.R.; FERREIRA, V.L.P. Atividade de polifenoloxidase e alterações da cor e dos teores de taninos condensados em novas cultivares de feijão (Phaseolus vulgaris L.) durante o armazenamento. Coletânea ITAL, v.19, p.154-164, 1989.

LAJOLO, F.M.; GENOVESE, M.I.; MENEZES, E.W. Qualidade nutricional. In: ARAUJO, R.S.; RAVA, C.A.; STONE, L.F.; ZIMMERMANN, M.J.O. (Coord.). Cultura do feijoeiro comum no Brasil. Piracicaba: Potafos, 1996. p.23-56.

LAM-SANCHEZ, A.; DURIGAN, J.F.; CAMPOS, S.L.; SILVESTRE, S.R.; PEDROSO, P.A.C.; BANZATTO, D.A. Efeitos da época de semeadura sobre a composição química e características físico-químicas de grãos de Phaseolus vulgaris L., Phaseolus angularis (Wild) Wright e Vigna unguiculata (L.) Walp. Alimentos e Nutrição, v.2, p.35-44, 1990.
MORENO, C.R.; LOPEZ, O.P. Endurecimento del frijol común. Cuadernos del Nutricion, v.15, p.17-32, 1992.

PÁRRAGA, M.S.; JUNQUEIRA NETTO, A.; PEREIRA, P.; BUENO, L.C.S.; PENONI, J.S. Avaliação do conteúdo de proteína total de duzentas cultivares de feijão (Phaseolus vulgaris L.) visando seu melhoramento genético. Ciência e Prática, v.5, p.7-17, 1981.

PIMENTEL, M.L.; MIRANDA, P.; COSTA, A.F.; MIRANDA, A.B. Estudo nutricional de linhagens de feijão comum (Phaseolus vulgaris L.). Revista Brasileira de Sementes, v.10, p.55-65, 1988.

POMPEU, A.S. Feijão. In: FURLANI, A.M.C.; VIÉGAS, G.P. (Ed.). O melhoramento de plantas no Instituto Agronômico. Campinas: Instituto Agronômico, 1993. p.111-155.

RAMOS JÚNIOR, E.U. Componentes do rendimento, qualidade de sementes e características tecnológicas em cultivares de feijoeiro. 2002. 72p. Dissertação (Mestrado) - Universidade do Estado de São Paulo, Botucatu.

SARRUGE, J.R.; HAAG, H.P. Análises químicas em plantas. Piracicaba: Esalq, 1974. 56p.

SAWAZAKI, H.E.; TEIXEIRA, J.P.F.; MORAES, R.M.; BULISANI, E.A. Modificações bioquímicas e físicas em grãos de feijão durante o armazenamento. Bragantia, v.44, p.375-390, 1985.

VIEIRA, R.F.; ROMEIRO, E.M.C.; SOUZA, L.R.P.; DONZELLI, M.F.; VIEIRA, V. Tempo de cocção, rendimento alimentar e aceitabilidade de feijões secos dos gêneros Vigna e Phaseolus. Revista Ceres, v.36, p.525-537, 1989.

YOKOYAMA, L.P.; BANNO, K.; KLUTHCOUSKI, T. Aspectos socioeconômicos da cultura. In: ARAUJO, R.S.; RAVA, C.A.; STONE, L.F.; ZIMMERMANN, M.J.O. (Coord.). Cultura do feijoeiro comum no Brasil. Piracicaba: Potafos, 1996. p.2-4.

ZIMMERMANN, M.J.O.; CARNEIRO, J.E.S.; DEL PELOSO, M.J.; COSTA, J.G.C.; RAVA, C.A.; SARTORATO, A.; PEREIRA, P.A.A. Melhoramento genético e cultivares. In: ARAUJO, R.S.; RAVA, C.A.; STONE, L.F.; ZIMMERMANN, M.J.O. (Coord.). Cultura do feijoeiro comum no Brasil. Piracicaba: Potafos, 1996. p.223-273.

Recebido em 29 de setembro de 2003 e aprovado em 22 de dezembro de 2003 